\title{
Chronic Hand Swelling and Dactylitis in Leprosy: A Case Report and Review of the Literature
}

\author{
Sonia Gupta ${ }^{1}$, Changzhao Li $^{2}$, Vinay Kumar Thallapally ${ }^{1}$, Poonam Sharma ${ }^{2}$, Joseph Nahas ${ }^{3}$ \\ 1. Internal Medicine, Creighton University, Omaha, USA 2. Pathology, Creighton University, Omaha, USA 3. \\ Rheumatology, Creighton University, Omaha, USA
}

Corresponding author: Sonia Gupta, drsoniagh1@gmail.com

\begin{abstract}
Leprosy is an infectious disease that is associated with various types of presentations. Diagnosis of the disease can be tricky in cases of atypical presentations. We report a unique case of leprosy characterized by chronic hand swelling, dactylitis, and seropositive laboratory markers, which was diagnosed in a rheumatology clinic.
\end{abstract}

Categories: Dermatology, Pathology, Rheumatology

Keywords: leprosy, erythema nodosum leprosum, rheumatism, autoantibodies

\section{Introduction}

Hansen's disease (leprosy) is an infectious disease that affects the skin, peripheral nerves, upper respiratory tract, and eyes. Musculoskeletal involvement is common and the prevalence of arthritis in leprosy ranges from 1-78\% [1]. Hence, it is important for rheumatologists to be familiar with the various aspects of this condition. Leprosy can have autoantibodies, and the topic of the prevalence of autoantibodies in patients with leprosy is a controversial one. It is usually diagnosed clinically. Diagnosis can be challenging in cases with non-specific symptoms, especially rheumatic symptoms, which are often referred to a rheumatology clinic. Through this case report, we attempt to highlight and analyze leprosy with rheumatologic symptoms as the initial presentation.

\section{Case Presentation}

A 24-year-old female, an immigrant, presented with swelling of the face and bilateral hands of one month's duration. She had initially visited her primary care physician for painful swelling over the small joints of both the hands and feet along with painful rash over the arms and legs of one month's duration. She also had intermittent fever and morning stiffness. Laboratory workups were ordered with autoimmune disorders as the main differential. Blood workup showed normocytic normochromic anemia. Inflammatory markers including erythrocyte sedimentation rate (ESR) and C-reactive protein (CRP) were found to be elevated. Regarding autoantibodies, anti-nuclear antibody (ANA), anti-cyclic citrullinated peptide (CCP), anticardiolipin (ACL), and beta 2 glycoprotein were positive, while rheumatoid factor (RF), SS-A/Ro

Review began 01/31/2021 Review ended 02/15/2021 Published 02/20/2021

\section{(c) Copyright 2021}

Gupta et al. This is an open access article distributed under the terms of the Creative Commons Attribution License CC-BY 4.0., which permits unrestricted use, distribution, and reproduction in any medium, provided the original author and source are credited. autoantibody, SSB/La autoantibody, anti-Smith (Sm) antibody, anti-double-stranded DNA (anti-dsDNA) antibodies, anti-Jo-1 antibody, and anti-centromere and perinuclear anti-neutrophil cytoplasmic antibodies (p-ANCA) were negative. Complement 3 (C3) and complement (C4) were normal (Table 1). X-ray of the bilateral hands was unremarkable. The patient was started on oral steroids and referred to a rheumatology clinic. On presentation to the rheumatology clinic, her face, hand, and feet were puffy with tenderness over small joints (Figure 1, Figure 2). There was a vague, ill-defined patch over the forehead and tender hyperpigmented nodules on the arm and legs. Peripheral nerves were not enlarged. Muscle strength was normal. The sensation was decreased on the lateral side of the left hand. She also had blunting of fine touch and pain sensation in the distal extremities. Clinical differential diagnosis included various rheumatologic diseases including rheumatoid arthritis. Skin biopsy of the painful nodules from the arm was performed on the same day. 


\section{Cureus}

\section{Variables}

White blood count

Hemoglobin

ESR

C-reactive protein

Anti-CCP

RF

ANA

Anti-dsDNA antibody

Anti Jo-1 immunoglobulin G

Anti-Sm antibody

p-ANCA

Anti-beta glycoprotein immunoglobin M

Anti-beta glycoprotein immunoglobulin G

ACL immunoglobulin G

ACL immunoglobulin M

C3

C4

25-hydroxy vitamin D

\section{At initial encounter}

8.2

10.1

114

13.40

26

$<10$

Positive (titer was not done)

1

$<0.2$

0

Negative

112

$<10.1$

$<9$

$>150$

154

25

24
Reference range

4-12 k/uL

$12-16 \mathrm{~g} / \mathrm{dL}$

$0-25 \mathrm{~mm} / \mathrm{hr}$

$<9 \mathrm{mg} / \mathrm{L}$

0-19 units

$<15 \mathrm{IU} / \mathrm{ml}$

0-4 IU/ml

0-9 IU/ml

$0-40 \mathrm{AU} / \mathrm{ml}$

$<=20 \mathrm{U} / \mathrm{ml}$

$<=20 \mathrm{U} / \mathrm{ml}$

0-14 units

0-12 units

$90-180 \mathrm{mg} / \mathrm{dl}$

$15-40 \mathrm{mg} / \mathrm{dl}$

$30-80 \mathrm{ng} / \mathrm{dl}$

\section{TABLE 1: Laboratory markers including autoantibodies at the initial encounter}

ESR: erythrocyte sedimentation rate; CRP: C-reactive protein; ANA: antinuclear antibody; anti-CCP: anti-cyclic citrullinated peptide; ACL: anticardiolipin; RF: rheumatoid factor; anti-Sm: anti-Smith; anti-dsDNA: anti-double-stranded DNA; p-ANCA: perinuclear anti-neutrophil cytoplasmic antibodies; C3: complement 3; C4: complement 4 


\section{Cureus}

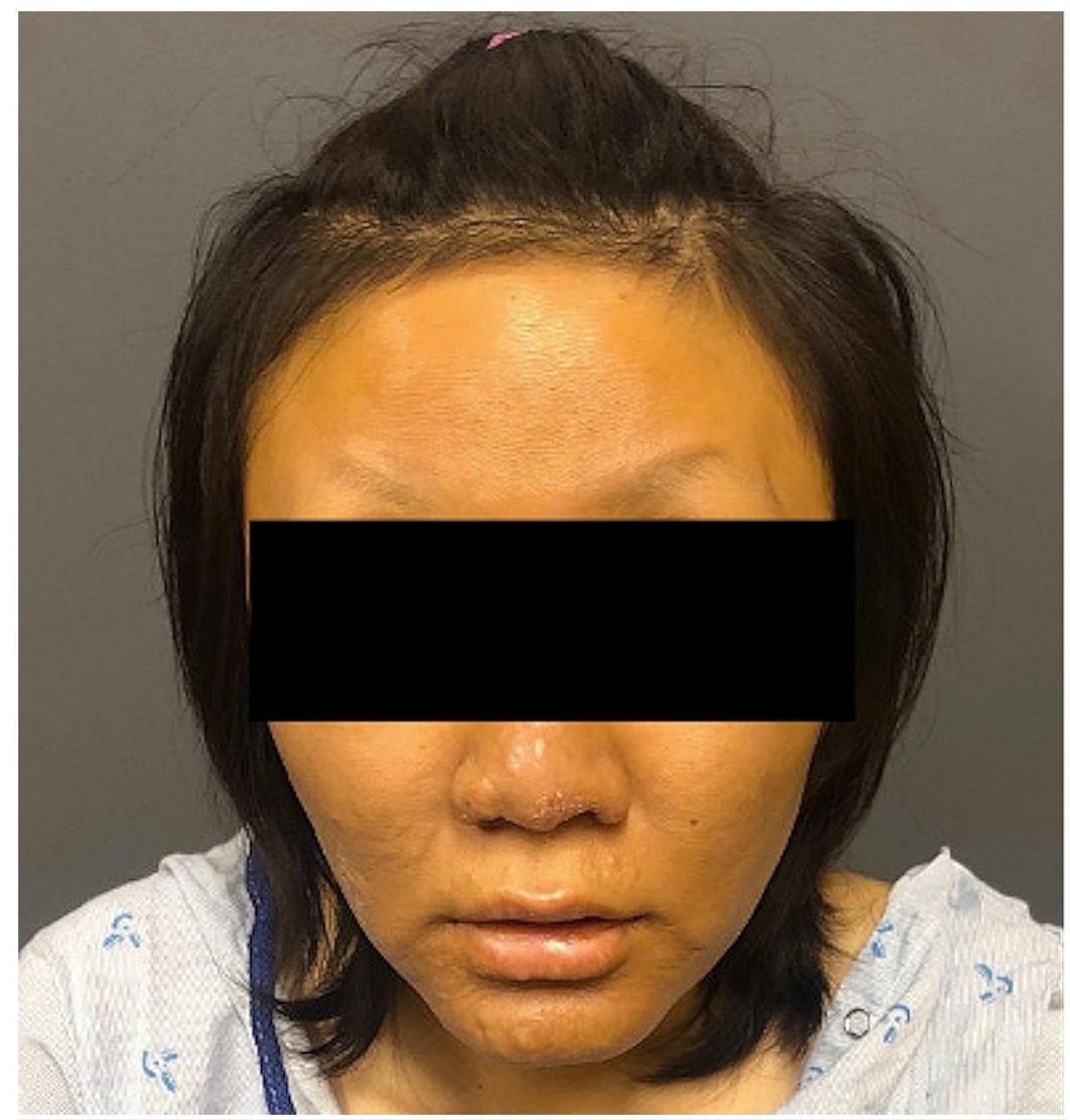

FIGURE 1: Picture showing facial swelling and loss of lateral eyebrows bilaterally 


\section{Cureus}

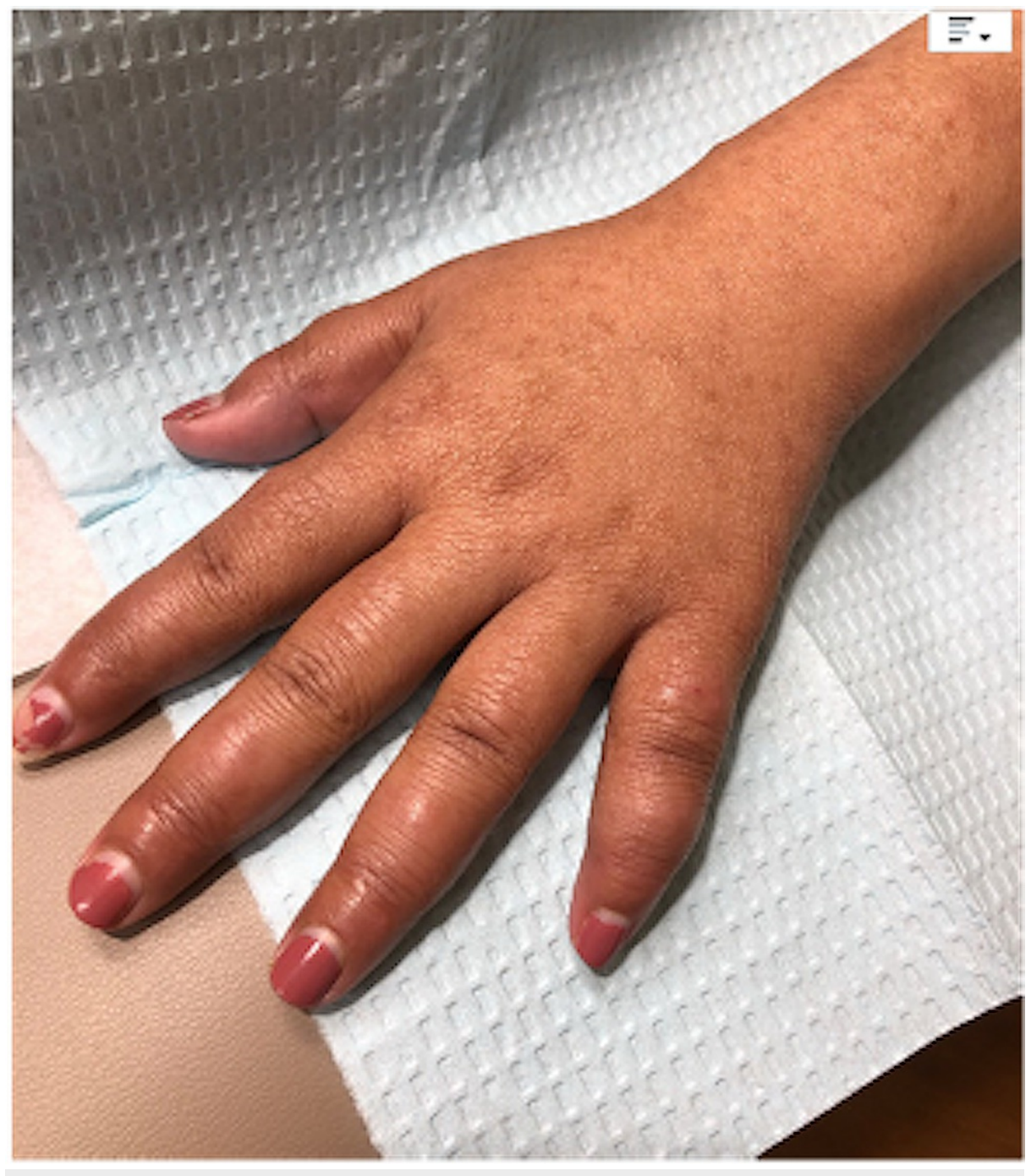

FIGURE 2: Swelling of joints of the left hand

Microscopically, the sections of the skin biopsy from the arm showed skin extending to the subcutaneous tissue. The sections showed acute and chronic inflammatory infiltrates replacing approximately $80-90 \%$ of the dermis and extending into the subcutaneous tissue forming lobular panniculitis (Figures $3 A, 3 B$ ). These infiltrates were composed of aggregates of foamy histiocytes with interspersed lymphocytes, eosinophils, and scattered neutrophils (Figures 3C, 3D, 3E) with surrounding blood vessels, adnexal structures, and cutaneous nerves at all levels of the dermis and subcutaneous tissue (Figures 3D, 3E, 3F). Within the reticular dermis, there were regions where dermal fibrosis intervened (Figures 3A, 3B). However, no vasculitis or fibrin thrombi were observed in the fat lobules and/or in the overlying dermis (Figures $3 C, 3 E$ ). Histiocytes within the inflammatory infiltrate showed prominent gray-blue granular substance within the cytoplasm (Figures 3D, 3F). Acid-fast Bacilli and Fite stains revealed large numbers of clumped, intact, as well as beaded acidfast bacilli within vacuolated histiocytes as well as within cutaneous nerves (Figures 3F, 3G, 3H).

Once the biopsy was back, a slit skin smear was performed in an outpatient setting to rule out leprosy. To assess the bacterial index (BI), air-dried slit skin smears were taken from both ear lobules, knee, forearm, and forehead. The slides were subjected to acid-fast staining with positive and negative controls according to the protocol of "Preparation and Examination of Skin Smears" published on the website of the National Hansen Disease Program (NHDP). To optimize the staining effects, alkaline methylene blue was stained for either one minute (Figures $4 A, 4 B, 4 C, 4 D$ ) or 30 seconds (Figures $4 E$, $4 F$ ). The stained smears were examined with a microscope using the oil immersion objective (x100) to determine the total number of bacilli. As shown in Figure 3, a large number of acid-fast bacilli were present in the form of globi (BI: $5+$ ) and were in the smears. Because of the rarity of leprosy in the state of Nebraska, the case was sent to the NHDP for an expert consult and confirmatory molecular test. Polymerase chain reaction (PCR) for Mycobacterium leprae (M. leprae) DNA was positive. The diagnosis of Hansen's disease [lepromatous (borderline/lepromatous) leprosy, active, consistent with type 2 reaction, erythema nodosum leprosum (ENL)] was finally made by the NHDP. The patient was started on minocycline, moxifloxacin, and rifampin once a month and her swelling has improved after one month of therapy. 


\section{Cureus}
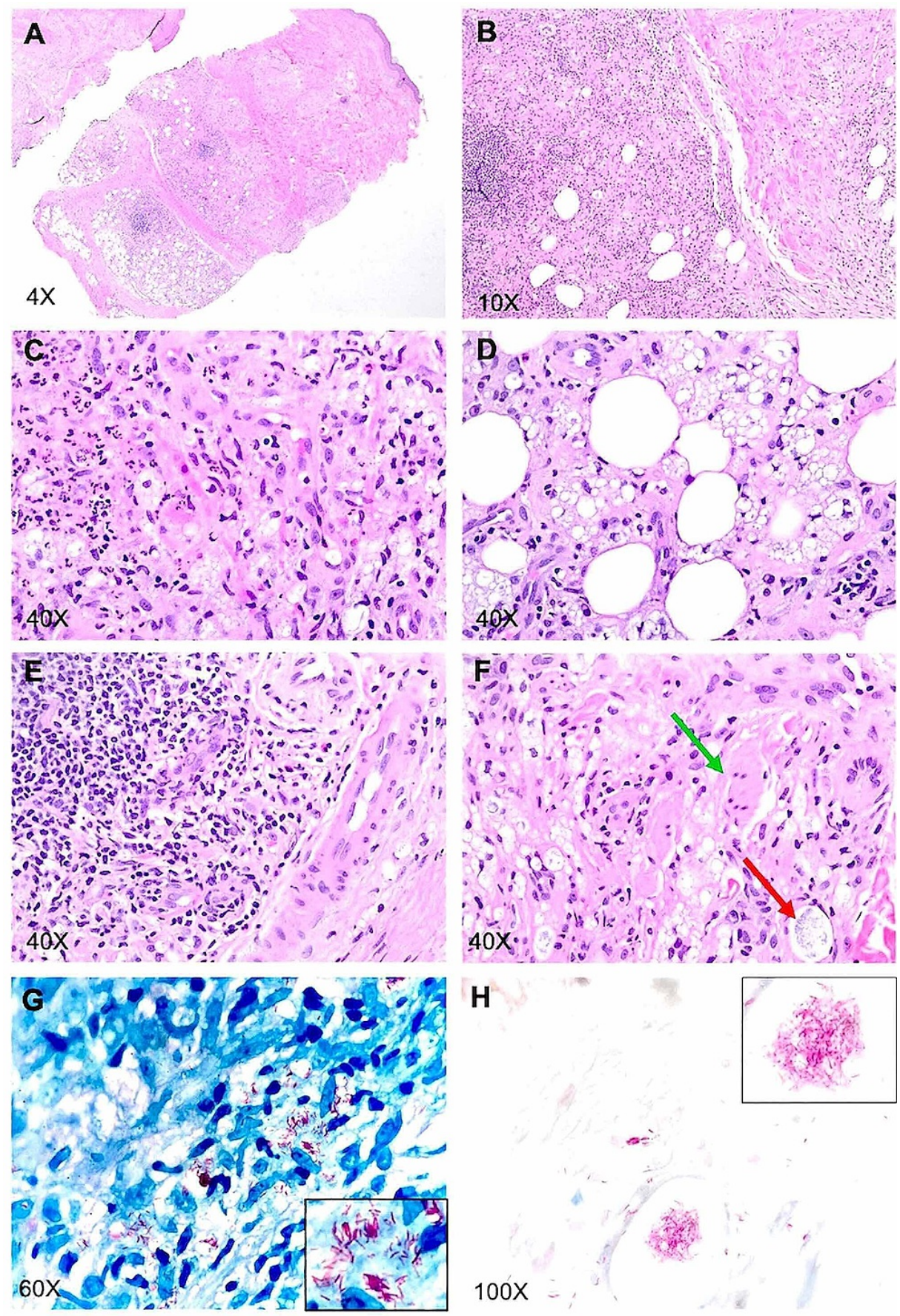

FIGURE 3: Pictures showing H\&E, Fite, and AFB staining of skin biopsy

A-F: H\&E staining. The red arrow indicates grayish granular material, which was proven to be $\mathrm{M}$. leprae globi following acid-fast stain. The green arrow indicates cutaneous never that is entrapped by foamy macrophages loaded with M. leprae. G: FITE staining. H: AFB staining 


\section{Cureus}
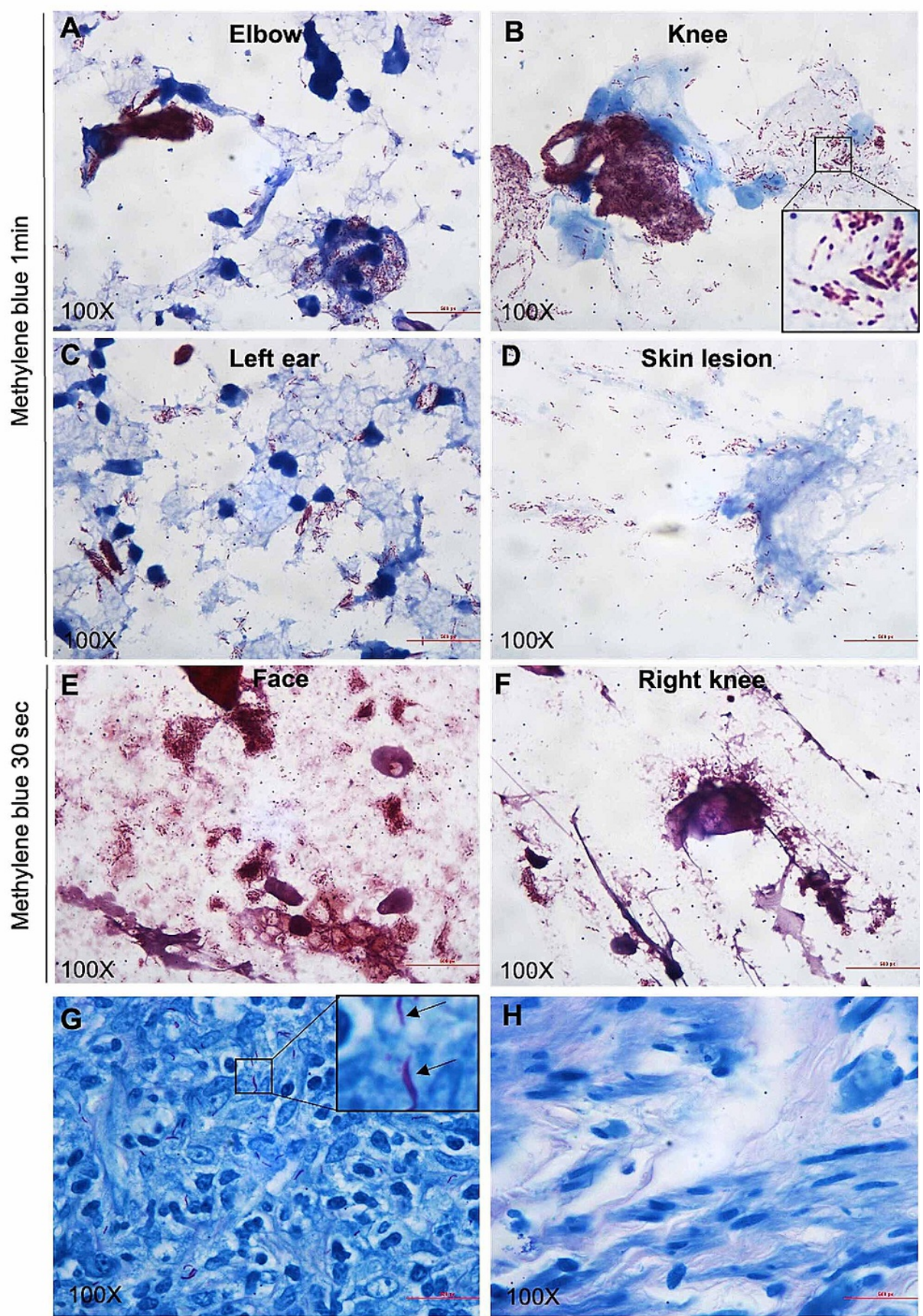

Positive control

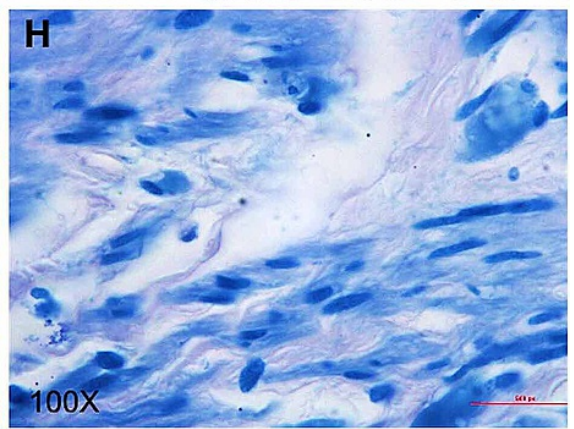

Negative control

FIGURE 4: Pictures showing Fite stain of slit skin smear from different locations

A-F: slit skin smear. G: positive control. H: negative control

\section{Discussion}

The diagnosis of leprosy can be challenging when rheumatological features constitute the initial presentation with minimal skin or nerve involvement. Musculoskeletal and autoantibodies in leprosy can mimic rheumatic diseases. Leprosy may present for the first time with inflammatory arthritis and is mistakenly treated with anti-rheumatic drugs with potentially disastrous consequences [2]. Inflammatory arthritis is commonly seen with lepromatous leprosy with or without lepra reactions [3]. Currently, no definite classification of arthritis exists. However, different forms of articular involvement in leprosy defined in the literature are Charcot joints, septic arthritis, polyarthritis of lepra reaction, and chronic arthritis [1]. The arthritis of lepra reaction is usually acute symmetrical, inflammatory, and polyarthritis affecting small joints of the hands and feet, which presents a picture similar to rheumatoid 
arthritis. In a cross-sectional study of 24,292 patients with ENL, $24 \%$ developed large joint arthritis, and $12 \%$ developed joint arthritis. Other extracutaneous manifestations were peripheral edema with $52.4 \%$, dactylitis with $13.7 \%$, and lymphadenitis with $14.7 \%$ [4]. The hand swelling in our case was likely due to tenosynovitis caused by M. leprae. The pathogenesis of articular involvement in leprosy is still elusive. Proposed mechanisms include intra-articular immune complex deposition and complement activation that could be involved in ENL-associated arthritis, direct bacilli infiltration of the synovium, or reactive arthritis to mycobacterium antigens [5].

The radiological features can range from normal to subluxation, periostitis, bone resorption, and complete destruction. Clinically painful cutaneous red nodules or plaques can be mistaken as ENL. Systemic symptoms including fever, malaise, and weight-loss as well as synovitis, arthritis, and dactylitis that our patient presented with are common manifestations of ENL [6]. Other rheumatologic manifestations reported include systemic lupus erythematosus (SLE)-like presentation and swollen hand and feet syndrome with remitting seronegative symmetrical synovitis with pitting edema [7], dermatomyositis-like presentation [8], enthesitis, sacroiliitis [1], and isolated tenosynovitis [9].

Leprosy can have autoantibodies and the topic of the prevalence of autoantibodies in patients with leprosy is a controversial one [10]. The frequent autoantibodies in leprosy are RF, ANA, anti-SS-B,

antimitochondrial, and antithyroglobulin [11]. A few studies showing the frequency of autoantibodies in leprosy patients are listed in Table 2 [12-17]. The seropositivity can make the diagnosis of leprosy complicated in patients with rheumatology presentation. It is noteworthy that studies have suggested vitamin D deficiency to be associated with increased autoimmune response in leprosy patients $[11,13]$. The prevalence of antiphospholipid in leprosy is highly variable and is mostly seen in the lepromatous form and as Lucio phenomenon, which clinically presents as painful, stellar lesions with an atrophic scar on the trunk and upper limbs and can mimic vasculitis [17].

ENL is an acute inflammatory reaction to M. leprae antigens, and it commonly occurs after treatment in patients with lepromatous leprosy (LL) or borderline leprosy. Histologically, it is characterized by lobular panniculitis with neutrophilic infiltrates superimposed on chronic multibacillary leprosy [18]. Lobular panniculitis with vasculitis can be seen in rheumatoid arthritis patients and patients with erythema induratum of Bazin (EIB) [18]. However, the neurotropic lymphohistiocytic infiltration with acid-fast bacilli is pathognomonic for Hansen's disease. In fact, both neurotropic granulomatous and lymph histiocytic infiltrate should point towards Hansen's disease or at least be considered in the differential diagnosis. Other soft signs of Hansen's disease include Grenz zone and Virchow cells (foamy macrophages), and the latter was also present in this case [19].

\begin{tabular}{|c|c|c|c|c|c|c|c|}
\hline Study & Sample size & Anti-CCP & RF & ANA & ACL & C-ANCA & Reference value \\
\hline Ribiero et al., 2008 & 185 & $2.6 \%$ & $1.6 \%$ & & & & 12 \\
\hline Guedes-Barbosa et al., 2008 & 64 & $16.4 \%$ & $15 \%$ & & & & 13 \\
\hline Zavala-Cerna et al., 2012 & 67 & $9.3 \%$ & $41.2 \%$ & & & & 14 \\
\hline Ribeiro et al., 2012 & 87 & & & $22 \%$ & & & 15 \\
\hline Neder et al., 2014 & 50 & & & & IgM; 16\%; IgG: 2\% & & 16 \\
\hline Pradhan et al., 2004 & 75 & & & & & $62.5 \%$ & 17 \\
\hline
\end{tabular}

\section{TABLE 2: Table illustrating studies with the percentage of autoantibodies seen in leprosy patients}

ANA: antinuclear antibody; anti-CCP: anti-cyclic citrullinated peptide; ACL: anticardiolipin; RF: rheumatoid factor; c-ANCA, cytoplasmic antineutrophil cytoplasmic antibody; IgM: immunoglobulin M; IgG: immunoglobulin G

In the reported case, some aspects drew our attention. Our patient was a young female with painful swelling of the fingers and positive anti-CCP and ANA that could indicate rheumatoid arthritis as the cause of the illness. Thorough skin and neurological examination, slit skin smear, and biopsy helped in our case diagnosis. Histologically, no vasculitis or fibrin thrombi were observed in the fat lobules and/or in the overlying dermis.

\section{Conclusions}

Our patient initially presented with rheumatologic features and seropositivity, which resulted in her being referred to the rheumatology clinic. Despite the rheumatologic manifestations, neurological findings and slit skin smear turned out to be clues for diagnosis. Several autoantibodies have been mentioned to be positive in such cases in the previously published literature, which was also the case with our patient, and can be 
misleading. Sometimes, rheumatological manifestation and seropositivity due to leprosy can be easily missed out and overlooked. A thorough clinical evaluation including skin and neurological examination is required to rule out leprosy. We would also like to raise awareness about considering leprosy as a differential diagnosis in arthritis and seropositivity as it will prevent the delay in the diagnosis and potentially devastating consequences with the early administration of immunosuppressants.

\section{Additional Information \\ Disclosures}

Human subjects: Consent was obtained or waived by all participants in this study. Conflicts of interest: In compliance with the ICMJE uniform disclosure form, all authors declare the following: Payment/services info: All authors have declared that no financial support was received from any organization for the submitted work. Financial relationships: All authors have declared that they have no financial relationships at present or within the previous three years with any organizations that might have an interest in the submitted work. Other relationships: All authors have declared that there are no other relationships or activities that could appear to have influenced the submitted work.

\section{Acknowledgements}

Li Changzhao is the first author of this article as well. I would like to thank Dr. Manasa Velagapudi and Dr. Christopher Huerter for their help in this case.

\section{References}

1. Chauhan S, Wakhlu A, Agarwal V: Arthritis in leprosy. Rheumatology (Oxford). 2010, 49:2237-2242. 10.1093/rheumatology/keq264

2. Salvi S, Chopra A: Leprosy in a rheumatology setting: a challenging mimic to expose . Clin Rheumatol. 2013, 32:1557-1563. 10.1007/s10067-013-2276-5

3. Paira SO, Roverano S: The rheumatic manifestations of leprosy. Clin Rheumatol. 1991, 10:274-276. 10.1007/BF02208689

4. Walker SL, Balagon M, Darlong J, et al.: ENLIST 1: An international multi-centre cross-sectional study of the clinical features of erythema nodosum leprosum. PLoS Negl Trop Dis. 2015, 9:e0004065. 10.1371/journal.pntd.0004065

5. Pereira HL, Ribeiro SL, Pennini SN, Sato EI: Leprosy-related joint involvement. Clin Rheumatol. 2009, 28:79-84. 10.1007/s10067-008-0986-X

6. Cossermelli-Messina W, Festa Neto C, Cossermelli W: Articular inflammatory manifestations in patients with different forms of leprosy. J Rheumatol. 1998, 25:111-119.

7. Helling CA, Locursio A, Manzur ME, Sormani de Fonseca ML: Remitting seronegative symmetrical synovitis with pitting edema in leprosy. Clin Rheumatol. 2006, 25:95-97. 10.1007/s10067-005-1145-2

8. Fernandes TR, Korinfskin JP, Espíndola MM, Corrêa LM: Arthritis and diagnosis of leprosy: a case report and review of the literature. An Bras Dermatol. 2014, 89:323-325. 10.1590/abd1806-4841.20142857

9. Al-Raqum HA, Uppal SS, El Abdalghani RA, Lasheen I: First report of leprosy presenting as acute polyarthritis in the setting of type I downgrading lepra reaction. Clin Rheumatol. 2006, 25:101-105. 10.1007/s10067-005-1147-0

10. Wright DJ: Autoantibodies in leprosy. Lancet. 1973, 2:40. 10.1016/s0140-6736(73)91971-5

11. El-Gendy H, El-Gohary RM, Shohdy KS, Ragab G: Leprosy masquerading as systemic rheumatic diseases. J Clin Rheumatol. 2016, 22:264-271. 10.1097/RHU.0000000000000379

12. Ribeiro SL, Pereira HL, Mangueira CL, Ferreira CE, Rosseto E, Scheinberg M: The development of arthritis and antinuclear antibodies correlate with serum 25-hydroxyvitamin D levels in patients with leprosy. Ann Rheum Dis. 2012, 71:2062-2063. 10.1136/annrheumdis-2012-201485

13. Guedes-Barbosa LS, Mangueira C, Scheinberg M: Anticitrulline peptide antibodies (CCP3) in leprosy sera: a negative association. Clin Rheumatol. 2008, 27:515-516. 10.1007/s10067-007-0810-z

14. Zavala-Cerna MG, Fafutis-Morris M, Guillen-Vargas C, Salazar-Páramo M, García-Cruz DE, Riebeling C, Nava A: Anti-cyclic citrullinated peptide antibodies and rheumatoid factor sera titers in leprosy patients from Mexico. Rheumatol Int. 2012, 32:3531-3536. 10.1007/s00296-011-2224-0

15. Ribeiro SL, Pereira HL, Silva NP, Neves RM, Sato EI: Anti-cyclic citrullinated peptide antibodies and rheumatoid factor in leprosy patients with articular involvement. Braz J Med Biol Res. 2008, 41:1005-1010. 10.1590/s0100-879x2008001100010

16. Neder L, Rondon DA, Cury SS, Silva CA: Musculoskeletal manifestations and autoantibodies in children and adolescents with leprosy. J Pediatr (Rio J). 2014, 90:457-463. 10.1016/j.jped.2014.01.007

17. Pradhan V, Badakere SS, Shankar Kumar U: Increased incidence of cytoplasmic ANCA (cANCA) and other autoantibodies in leprosy patients from western India. Lepr Rev. 2004, 75:50-56.

18. Gupta P, Saikia UN, Arora S, De D, Radotra BD: Panniculitis: a dermatopathologist's perspective and approach to diagnosis. Indian J Dermatopathol Diagn Dermatol. 2016, 3:29. 10.4103/2349-6029.195224

19. Fischer M: Leprosy - an overview of clinical features, diagnosis, and treatment . J Dtsch Dermatol Ges. 2017, 15:801-827. 10.1111/ddg.13301 